\title{
Tubercular mastitis - A case report with uncommon presentation
}

\author{
Prerna Aggarwal', Sushmita Sharma², Pradeep Singh ${ }^{3}$, Maninder Kaur ${ }^{1}$, Ashwini Manhas ${ }^{4}$, Manju Bala ${ }^{4}$ \\ ${ }^{1}$ Associate Professor, Deptartment of Microbiology, ${ }^{2}$ Associate Professor, Department of Obstetrics \& Gynae, ${ }^{3}$ Professor, Department of \\ Surgery, ${ }^{4}$ Assistant Professor, Deptartment of Microbiology, Gian Sagar Medical College \& Hospital affiliated to Baba Farid University of Health \\ Sciences, Punjab, India
}

\section{A B S T R A C T}

Tuberculosis of the breast is significant due to its rare occurrence and mistaken identity with breast cancer and pyogenic breast abscess. It typically affects young lactating multiparous women and can present either as an abscess or as a unilateral, painless breast mass. A case of 22 year unmarried female presented with discharge breast and a small abscess. Radiological and cytological methods remained nonspecific and tubercular mastitis was diagnosed on Zeihl Neelsen staining and culture for Mycobacteria. A multifaceted approach is needed to reach the correct diagnosis.
Access this article online

Website:

http://nepjol.info/index.php/AJMS

Key words: Tuberculosis, Breast, Discharge, Zeihl Neelsen staining

\section{INTRODUCTION}

Tuberculosis of the breast is significant due to its rare occurrence and mistaken identity with breast cancer and pyogenic breast abscess. The first case of breast tuberculosis was recorded by Sir Astley Cooper who described it as "scrofulous swelling of the bosom". ${ }^{1}$ Incidence of breast tuberculosis has been recorded to be as low as $0.1 \%$ of all breast diseases in developed countries and up to 3-4\% in developing countries like India and Africa. ${ }^{2}$ It typically affects young lactating multiparous women and can present either as an abscess or as a unilateral, painless breast mass. ${ }^{3}$ Although rare in the past after the development of antitubercular therapy, cases of breast tuberculosis along with other extra pulmonary sites has seen a rise due to AIDS cases and more conditions leading to immunocompromised states. Breast TB is paucibacillary and consequently tests such as microscopy, culture and nucleic acid amplification tests such as polymerase chain reaction techniques do not have the same diagnostic utility as they do in pulmonary tuberculosis. ${ }^{4}$ Thus, it is not uncommon for breast TB to be misdiagnosed either as non-specific abscess or carcinoma. ${ }^{5}$ Diagnosis of breast tuberculosis, therefore, remains a challenge for clinicians and requires a high degree of suspicion. The aim of this report was to highlight and emphasize the difficulty in diagnosis of a rare case of primary tuberculosis breast where microbiological and not radiological or histo-pathological examination was diagnostic.

\section{CASE REPORT}

A 22 years old, unmarried, well educated female visited surgery OPD in Dec'12 with chief complaint of lump breast. On examination, a lumpy mass was felt. On ultrasono-mammography, both breasts showed normal fibro glandular architecture. There was no evidence of mass/cysts in either breast, no e/o dilatation of ducts on either side, bilateral retroareolar region was normal, both axillae normal, no lymphadenopathy was seen. Aspiration for cytology was tried from the suspected site, but it showed fat cells only. The patient was sent back after symptomatic treatment. About a month later, the patient presented again, now to the Obstetrics and Gynae OPD with chief complaint of discharge from nipple right breast since one day. On examination, both breasts were normal with no lump on palpation, mild mastalgia and a minimal discharge was seen. The discharge was brownish in colour. There was no erythema and no retraction of nipple. Her menstrual history was normal and regular. All her routine investigations also showed no abnormality. Sono-mammography showed normal 
breasts. A surgical opinion was again sought for. Surgical consultation showed a small abscess with induration and brownish discharge right breast in peri-areolar region. The patient was advised for incision and drainage of the suspected lesion. She came for the procedure after about a month. Incision and drainage was done and the tissue was sent for histo-pathological examination which revealed chronic nonspecific inflammation. The patient was put on Augmentin and Lyser-D for 5 days.

After about a month of this procedure the patient presented again to the surgery OPD with similar complaint of discharge from right breast since one day and feeling of lump. There was no evidence or complaint of any other sign or symptom. She was investigated completely for haematological indices, blood glucose profile, thyroid function tests and chest X-ray. All reports were normal. A second time, the abscess was opened and incision with drainage and curettage was done. The tissue sent for histopathological examination and again showed non specific chronic inflammation. This time the abscess pus was sent for microbiological examination for gram staining, acid fast staining and bacterial cultures. Gram staining showed only polymorphs with no bacteria and bacterial culture was sterile upto $48 \mathrm{hrs}$. Initial screening under fluorescent microscope for acid fast bacilli was done followed by Zeihl Neelsen staining. Auramine staining (Figure 1) and the Zeihl Neelsen staining (Figure 2) both were positive for acid fast bacilli and later the culture on LJ medium was positive for Mycobacterium tuberculosis.

On the basis of smear examination by ZN staining, the patient was referred to DOTS centre for anti tubercular treatment. The patient was put on four drug (HRZE) therapy for two months and two drugs (HR) for four months, a total of six month therapy. The patient came

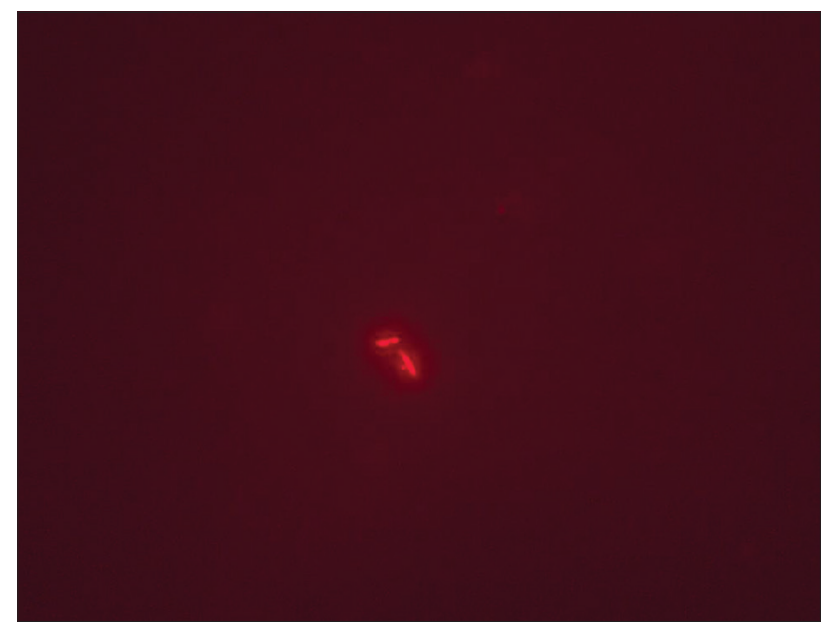

Figure 1: Photograph showing Mycobacteria on auramine staining under fluorescent microscope in the specimen from breast abscess of the patient for a follow up after three months and fine with no recurrence.

\section{DISCUSSION}

Tubercular mastitis is still considered a rare clinical entity as very few cases are reported. In India, approx. 10 million are affected by pulmonary tuberculosis but only a few hundred of breast tuberculosis. This can be attributed mainly to a lack of awareness of manifestation of this disease or misdiagnosis. ${ }^{6}$ The other reason is the resistance offered by breast tissue to the survival and multiplication of tubercle bacillus similar to that offered by spleen and skeletal muscle. ${ }^{7}$ Breast tuberculosis usually affects women in their reproductive age group of 20-40 yrs and is uncommon in pre-pubertal girls and older females. ${ }^{5}$ Females during lactation are more susceptible as lactating breast is more vascular and also susceptible to trauma. ${ }^{6}$ As contrast, our patient was a 22 year old unmarried female. A study conducted by Puneet et $\mathrm{al}^{5}$ showed incidence of breast tuberculosis as $4.13 \%$ out of 1016 breast lump cases out of which $19 \%$ were lactating, and $88 \%$ were misdiagnosed.

Tuberculosis of breast may be primary or secondary to lesion elsewhere in the body. Primary form is quite rare. ${ }^{8}$ In our case, as there was no other focus found in the body on complete examination, the lesion was primary.

The commonest presentation of breast tuberculosis is as a lump in the breast, that too in the upper outer quadrant of the breast as in carcinoma. ${ }^{9}$ But in our case the site was sub-areolar which is quite unusual and the presenting symptom was discharge from the nipple with lump breast.

Different modalities are helpful in diagnosis of breast tuberculosis like sono-mammography, cytological studies

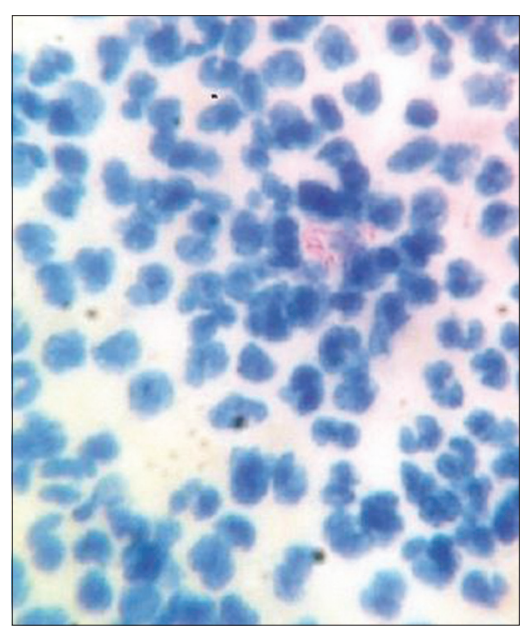

Figure 2: Photograph showing smear positive for acid fast bacilli from abscess aspirate 
and microbiological examination. In our case the first two modalities gave non specific results and the patient presented with repeated lesion. High degree of suspicion by the surgeon and microbiological examination led to the definite diagnosis by direct detection of the acid fast bacilli in Zeihl Neelsen staining and later by culture which is the gold standard for diagnosis. In breast abscess like picture dominated by acute inflammatory exudates, AFB positivity or histological confirmation is mandatory to call the lesion tubercular. ${ }^{10}$

As a conclusion, tubercular mastitis is still an uncommon entity and awareness of the disease is important. Diagnosis depends on the combined picture of all the diagnostic tests as it presents in unusual forms and any one diagnostic modality may overcome the limitations of the others. Therefore, the specimen should be examined as widely as possible.

\section{REFERENCES}

1. Longman, Rees, Orme, Brown and Green. A. Cooper Illustrations of the diseases of the breast., London (1829).
2. Luh SP, Chang KJ, Cheng JH, Hsu JD and Huang CS. Surgical treatment for primary mammary tuberculosis - report of three octogenarian cases and review of literature. Breast Journal 2008; 14: 311-312.

3. Tewari M and Shukla HS. Breast tuberculosis: diagnosis, clinical features and management. Indian $\mathrm{J}$ Med Res 2005;122:103-110.

4. Pai M, Riley LW and Colford JM. Interferon-gamma assays in the immune-diagnosis of tuberculosis: a systematic review. Lancet Infect Dis 2004; 4:761.

5. Puneet, S Tiwary, R Ragini, S Singh, S Gupta and V Shukla. Breast Tuberculosis: Still Common In India. The Internet Journal of Tropical Medicine 2004;2 (2).

6. Banerjee S N, Ananthakrishnan N, Mehta R B and Prakash S. Tuberculous mastitis: a continuing problem. World J Surg 1987; 11:105-109.

7. Hamit HF and Ragsdale TH. Mammary tuberculosis. J R Soc Med 1982;75; 764-765.

8. Zandrino F, Monetti F and Gandolfo N. Primary tuberculosis of the breast. A case report. Acta Radiologica 2000; 41:61-63.

9. Oh KK, Kim JH and Kook SH. Imaging of tuberculous disease involving breast. Eur Radiol 1998; 8: 1475-1480.

10. Kakkar S, Kapila K, Singh MK and Verma K. Tuberculosis of the Breast: A cyto-morphologic study. Acta Cytol 2000;44;292-296.

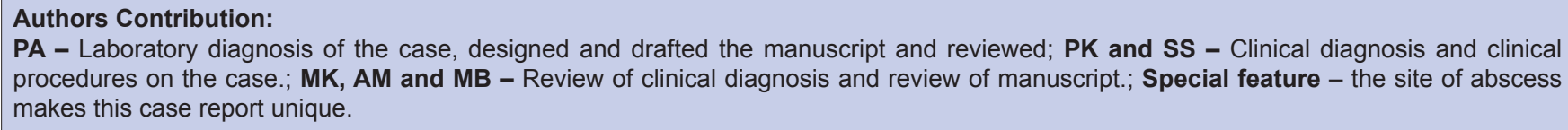

Source of Support: Nil, Conflict of Interest: None declared. 